How to determine optical gaps and voltage losses in organic photovoltaic materials

Peer-reviewed author version

VANDEWAL, Koen; Benduhn, J. \& Nikolis, V. C. (2018) How to determine optical gaps and voltage losses in organic photovoltaic materials. In: SUSTAINABLE ENERGY \& FUELS, 2(3), p. 538-544.

DOI: $10.1039 / \mathrm{c} 7 \mathrm{se} 00601 \mathrm{~b}$

Handle: http://hdl.handle.net/1942/28691 


\section{How to determine optical gaps and voltage losses in organic photovoltaic materials}

Received 00th January 20xx Accepted 00th January 20xx

DOI: $10.1039 / \times 0 x \times 00000 x$

www.rsc.org/

\author{
K. Vandewal, ${ }^{a, b}$ J. Benduhn ${ }^{a}$ and V. C. Nikolis ${ }^{a}$
}

The best performing organic solar cells (OSC) efficiently absorb photons and convert them to free charge carriers, which are subsequently collected at the electrodes. However, the energy lost in this process is much larger than for inorganic and perovskite solar cells, currently limiting the power conversion efficiency of OSCs to values slightly below $14 \%$. To quantify energy losses, the open-circuit voltage of the solar cell is often compared to its optical gap. The latter is, however, not obvious to determine for organic materials which have broad absorption and emission bands, and is often done erroneously. Nevertheless, a deeper understanding of the energy loss mechanisms depends crucially on an accurate determination of the energies of the excited states involved in the photo-conversion process. This perspective therefore aims to summarize how the optical gap can be precisely determined, and how it relates to energy losses in organic photovoltaic materials.

\section{Introduction}

In the past 15 years, the power conversion efficiency (PCE) of organic solar cells (OSCS) has increased from about $1 \%$ to now almost $14 \%{ }^{1}$ This development has been accomplished by the synthesis of new electron donating and electron accepting materials of which high performing combinations have been discovered. The highest external quantum efficiencies (EQE $E_{P V}$ ) exceed $80 \%,^{2}$ and fill-factors (FF) approach $80 \%{ }^{3}$ being on-par with those of higher efficiency technologies, such as gallium arsenide (GaAs) and crystalline silicon (c-Si). Up to now, it is the open-circuit voltage of OSCs ( $\left.V_{O C}\right)$ which is lagging behind, making OSCs currently still less efficient than established inorganic photovoltaic technology or the emerging perovskite solar cells. Therefore, research is nowadays focusing on the identification of the elementary processes responsible for the large difference between $e V_{o c}$ and the optical gap of the main absorber $\left(E_{\text {opt }}\right)$, where $e$ is the elementary charge. At the same time, new materials are being synthesized, ${ }^{4}$ as well as new device architectures have been developed, ${ }^{5}$ with the aim to minimize these voltage losses.

Under solar illumination, the total energy loss per absorbed photon is equal to the difference between the photon energy and the product of $e$ with the voltage at the point of maximum electrical power output. A lower limit for these energy losses is given by the difference between the device's optical gap $\left(E_{\mathrm{opt}}\right)$ and $e V_{\mathrm{Oc}}$. Indeed, the potential energy of the extracted charge carriers is limited to $e V_{\mathrm{oc}}$, while $E_{\mathrm{opt}}$ is given by the lowest energy singlet exciton, either of the donor $\left(E_{\mathrm{D}^{*}}\right)$ or the acceptor $\left(E_{\mathrm{A}^{*}}\right)$. For the remainder of this perspective, we refer to $E_{\mathrm{opt}} / e$ - Voc as "voltage loss".

To precisely characterize and study energy and voltage loss mechanisms for OSCs, an accurate determination of the energies of the relevant electronic states in organic semiconductors is crucial. Following photon absorption, several electron-transfer steps introduce energy losses. Figure 1 depicts the energy levels of singlet and triplet states on the neat donor (D) and acceptor (A) in a Jablonski diagram. Following electron transfer, an intermolecular charge-transfer (CT) state, comprising an electron on the acceptor and a hole on the donor, is formed. This state can decay to the ground state or dissociate into a state comprising fully free charges (FC).

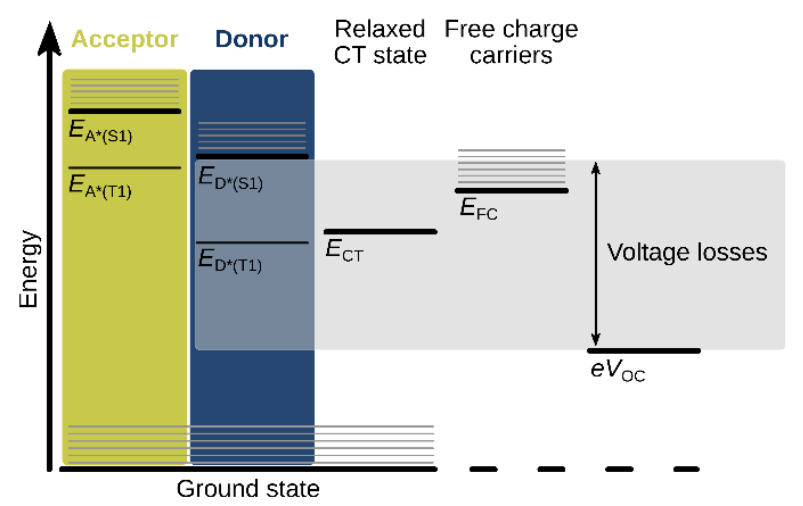

Figure 1: Jablonski diagram, showing the energy levels which are important for OSCS. The lowest optical excitations in the absorber molecules, i.e. $A$ and $D$, are singlet excitons $\left(E_{\mathrm{A}^{*}(S 1)}\right.$ and $\left.E_{\mathrm{D}^{*}(\mathrm{~S} 1)}\right)$. The optical transition from the ground state to the triplet excited state $\left(E_{\mathrm{A}^{*}(T 1)}\right.$ and $\left.E_{D^{*}(T 1)}\right)$ are forbidden. The intermolecular CT state between $D$ and $A$ has the energy $E_{\mathrm{CT}}$. In OSCs charge generation and recombination takes place via this state, therefore, we can define voltage losses with respect to this state, or with respect to the strongly absorbing singlet states on the neat materials. The energy difference between the lowest energy singlet exciton and $e V_{o c}$ forms a lower limit for the energy lost in the conversion of strongly absorbed photons to charges collected at the electrodes.

\footnotetext{
a. Dresden Integrated Center for Applied Physics and Photonic Materials (IAPP) and Institute for Applied Physics, Technische Universität Dresden, Nöthnitzer Straße 61, 01187 Dresden, Germany. E-mail: koen.vandewal@iapp.de

b. Current address: Instituut voor Materiaalonderzoek (IMO), Hasselt University, Wetenschapspark 1, BE-3590, Diepenbeek, Belgium. E-mail: koen.vandewal@uhasselt.be
} 
The energy and voltage losses in OSCs relate to the chemical potential of the free charge carriers under solar illumination, which is determined by the energies of the relevant excited states, as well as the transition rates between these states and the ground state. However, the excited state energies are often empirically determined. For example, CT state energies are sometimes estimated by taking the difference between the energy of the highest occupied molecular orbital of the donor, $\mathrm{HOMO}(D)$, and lowest unoccupied molecular orbital of the acceptor, LUMO(A). This approach however, neglects polarization and binding energies, ${ }^{6}$ and values for the driving force for electron transfer can be over- or underestimated by several tenths of electronvolts $(\mathrm{eV})$. The singlet energies $E_{\mathrm{A}^{*}(\mathrm{~S} 1)}$ or $E_{\mathrm{D}^{*}(\mathrm{~S} 1)}$ of acceptor and donor, of which the lowest one can be considered as the optical gap $\left(E_{\text {opt }}\right)$ of the blend, are often taken as the onset of the absorption spectrum, which is rather ill- defined. It is clear that further progress in the fundamental understanding of OSC operation needs an unambiguous method to determine voltages losses, so that they can be compared between research groups. This perspective aims therefore to summarize how optical gaps and CT state energies can be consistently determined and how they relate to voltage losses in OSCs. a)

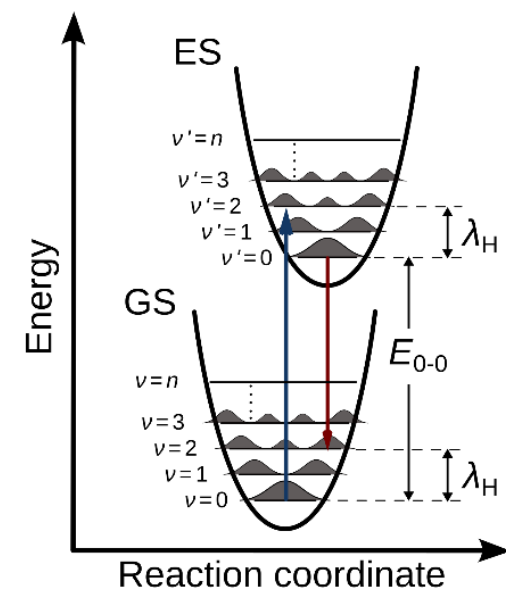

High frequency vibrations

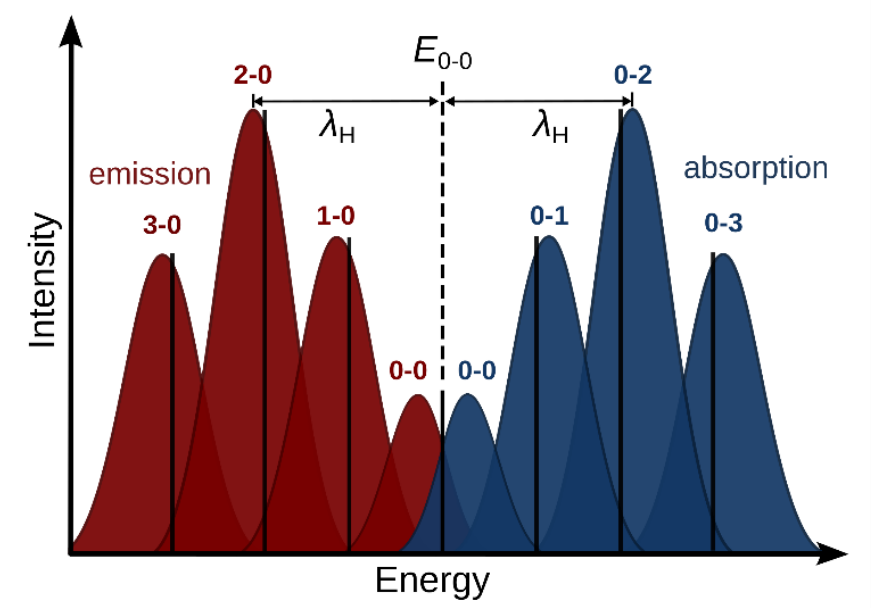

b)

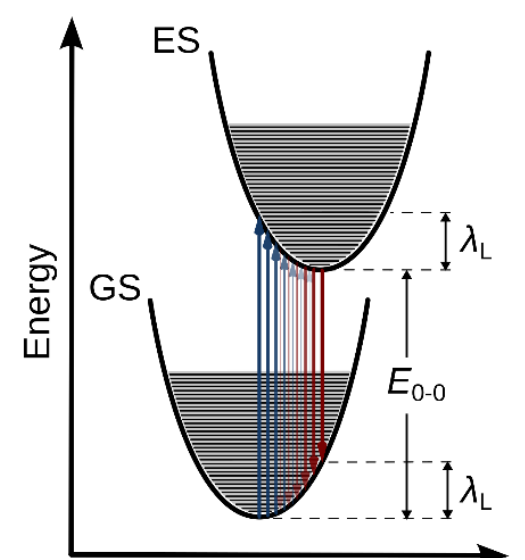

Low frequency vibrations

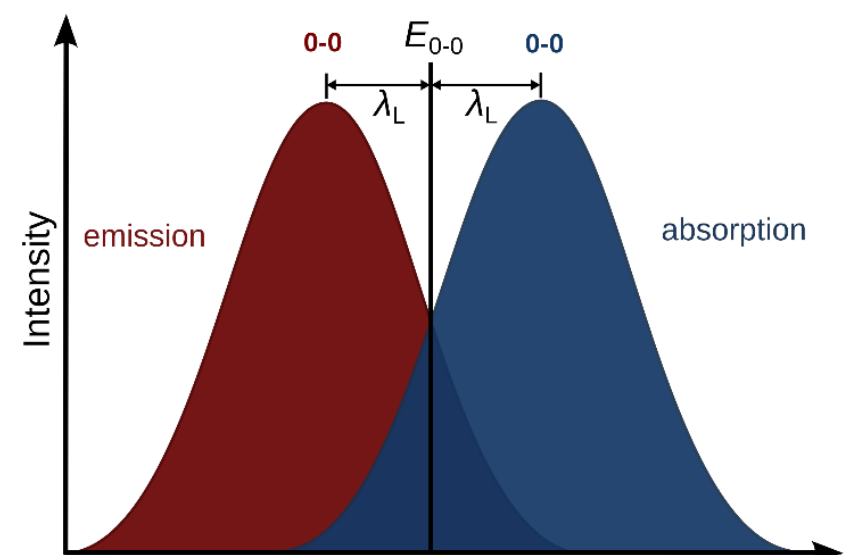

Figure 2: Optical transitions depicted in an energy diagram with displaced potential wells for the ground state (GS) and excited state (ES), taking into account that the reaction coordinate remains invariant during the transition (Franck-Condon principle). Vertical blue arrows represent absorption and vertical red arrows emission. (a) Absorption and emission spectra are dominated by high frequency vibrations, with a relaxation energy $\lambda_{H}$. In this case, the spacing between vibrational levels is larger than the thermal energy. Photon absorption occurs by promoting an electron from the vibrational ground state level $v=0$ to an excited state vibrational level $v^{\prime}$, indicated by the black lines in the corresponding absorption spectrum. In the emission spectrum, transitions from the lowest excited state vibrational level $v^{\prime}=0$ to ground state levels $v$ dominate, as indicated by the black lines in the emission spectra. Absorption and emission spectra overlap around the 0-0 transition energy $\left(E_{0-0}\right)$. (b) Peak broadening by low frequency vibrations with reorganization energy $\lambda_{\mathrm{L}}$. The spacing between vibrational levels is less than the thermal energy. Optical transitions from higher energy thermally populated levels results in absorption at photon energies below $E_{0-0}$ and emission at energies above $E_{0-0}$. The resulting peak width depends on temperature and $\lambda_{\mathrm{L}}$. 


\section{Absorption, emission and the optical gap}

\section{Spectral broadening in organic materials}

For a hypothetical solar cell with an ideal step-wise absorption spectrum, determination of $E_{\mathrm{opt}}$ is trivial, as it corresponds to the lowest energy for which photons are strongly absorbed. In reality, OSCs exhibit rather shallow absorption tails, due to the presence of static and dynamic disorder, as well as the presence of weakly absorbing CT states. In order to unambiguously determine $E_{\mathrm{opt}}$, it is instructive to consider the origins of the spectral broadening.

The absorption and emission spectra of organic materials are strongly affected by electron-phonon coupling and molecular vibrations. Figure 2a shows how the high frequency vibrations, for example ring breathing modes, are responsible for discrete peaks in the spectra. ${ }^{7}$ For these high frequency modes the spacing between the vibrational energy levels is much larger than the thermal energy and electrons populate solely the lowest energy vibrational level $v=0$. Photon absorption and emission solely occurs for discrete photon energies related to transitions between a vibrational level of the ground state $v$, and a vibrational level of the excited state $v^{\prime}$. The 0 0 transition occurs at the energy $E_{0-0}$, which is the difference between vibrationally relaxed ground- and excited state.

However, the absorption and emission spectra of organic thin films seldom consist of well resolved, discrete peaks: A main source of peak broadening are low frequency vibrations with an energy spacing less than the thermal energy (Figure 2b). ${ }^{7}$ Optical transitions from thermally (Boltzmann) populated low frequency vibrational modes result in absorption at photon energies below $E_{0-0}$ and emission at photon energies above $E_{0-0}$. Treating the low frequency vibrations as harmonic oscillators results in Gaussian absorption $(A(E))$ and emission $(N(E))$ line-shapes

$$
\begin{aligned}
& A(E) \sim E \exp \left(-\frac{\left(E-E_{0-0}-\lambda_{L}\right)^{2}}{4 \lambda_{L} k_{\mathrm{B}} T}\right) \\
& N(E) \sim E^{3} \exp \left(-\frac{\left(E-E_{0-0}+\lambda_{L}\right)^{2}}{4 \lambda_{L} k_{\mathrm{B}} T}\right)
\end{aligned}
$$

Hereby, $E$ corresponds to the photon energy and $k_{\mathrm{B}}$ is the Boltzmann constant. The line-width is proportional to the temperature $T$ and the low frequency relaxation energy $\lambda_{\mathrm{L}}$. The energy difference between vibrational relaxed ground- and excited state, $E_{0-0}$, is the crossing point of the appropriately normalized absorption and emission spectra, as shown in Figure $2 b$.

\section{Extracting singlet and charge-transfer state energies}

In what follows, we outline how equations (1) and (2) can be used to accurately extract the energy levels $E_{\mathrm{D}^{*}(\mathrm{~S} 1)}$ or $E_{\mathrm{A}^{*}(\mathrm{~S} 1)}$ and $E_{\mathrm{CT}}$ for OSC blends. Hereby, we consider that the absorption spectrum of a donor-acceptor blend for OSCs will be mainly determined by high oscillator strength optical transitions on the neat materials. However, within their gap, much weaker absorption related to direct excitation of CT states is present. The optical gap $E_{\text {opt }}$ is identified as the $E_{0-0}$ transition energy of the singlet localized on either donor or acceptor. The $E_{0-0}$ of the CT state $\left(E_{\mathrm{CT}}\right)$ is often lower than $E_{\mathrm{opt}}$. Both
$E_{\mathrm{opt}}$ and $E_{\mathrm{CT}}$ can be determined from the optical spectra by a fit of the low energy absorption tail or high energy emission tail with Eq. (1) or Eq. (2), respectively. Alternatively, one can make use of the fact that $E_{0-0}$ coincides with the energy at which the appropriately normalized absorption and emission spectrum cross. Dividing $A(E)$ by $E$ and $N(E)$ by $E^{3}$, yields the so-called reduced absorption and emission spectra. When normalizing the reduced spectra to the maximum of the corresponding peak, Eq. (1) and Eq. (2) intersect exactly at $E=E_{0-0}$. In the case of mirror-image spectra, $E_{0-0}$ is the midpoint between the absorption and emission maxima (Stokes shift), separated by $2 \lambda_{L} \cdot{ }^{8}$

As a concrete example, Figure 3a shows the determination of $E_{\mathrm{opt}}$ $\left(E_{\mathrm{D}^{*}\left(\mathrm{~S}_{1}\right)}\right)$ and $E_{\mathrm{CT}}$ for vacuum-processed OSCs based on $\mathrm{ZnPc}: \mathrm{C}_{60}$ and $\mathrm{F}_{4}-\mathrm{ZnPc}: \mathrm{C}_{60}$ active layers. Photovoltaic diodes comprising both neat donors and their blends with $\mathrm{C}_{60}$ were fabricated. The electroluminescence (EL) and $\mathrm{EQE}_{\mathrm{PV}}$ spectra of these diodes were measured and provide a reliable measurement of the emission and absorption tails. It should be noted that EQEPV and absorption spectra are interchangeable here, since the internal quantum efficiency (IQE) of D:A photovoltaic blends is rather constant at their low energy tail. ${ }^{9}$ Furthermore, EL spectra were measured at low injection currents, keeping the system in quasi-equilibrium. ${ }^{9}$ For the neat phthalocyanines, the crossing point between the normalized reduced absorption (EQEPV) and reduced emission (EL) spectra yields a similar value for $E_{\mathrm{opt}}\left(\mathrm{E}_{\mathrm{D}^{*}\left(\mathrm{~S}_{1}\right)}\right)$ around 1.52-1.53 eV. For the blends with $\mathrm{C}_{60}$, reduced $\mathrm{EQE}_{\mathrm{PV}}$ and $\mathrm{EL}$ curves are plotted on a logarithmic scale, on which for $\mathrm{ZnPc}: \mathrm{C}_{60}$, an additional $\mathrm{CT}$ absorption band becomes visible. The EL spectrum of the blend is dominated by CT emission. Fits of EQEpv and EL with respectively Eq. (1) and (2) yield similar values for $E_{\mathrm{CT}}$, which coincide rather well with the crossing point of the spectra at $1.17 \mathrm{eV}$. Fluorination of the phthalocyanine donor leads to a decrease of both its HOMO and LUMO. Therefore, we observe a blue-shift of the $\mathrm{CT}$ absorption band for $\mathrm{F}_{4}-\mathrm{ZnPc}: \mathrm{C}_{60}$ blend as compared to $\mathrm{ZnPc}: \mathrm{C}_{60}$. In fact, the $\mathrm{CT}$ absorption becomes indistinguishable from the neat $\mathrm{F}_{4}-\mathrm{ZnPc}$ absorption tail. A fit of the $\mathrm{EL}$ spectrum with Eq. (2) and the crossing point between reduced $E L$ and EQE $_{\mathrm{pV}}$ yield an $E_{0-0}$ energy of $1.46 \mathrm{eV}$, i.e. $60 \mathrm{meV}$ lower than the $E_{\mathrm{opt}}$ of $\mathrm{F}_{4}-\mathrm{ZnPc}$. In this case optical transitions related to $\mathrm{CT}$ state excitation and low energy $\mathrm{F}_{4}$-ZnPc excitation are indistinguishable.

With the knowledge of $E_{\mathrm{opt}}$ and $E_{\mathrm{CT}}$ for both material systems, we can now perform a detailed analysis of the voltage losses, summarized in Figure $3 \mathrm{c}$ and $3 \mathrm{f}$. The $V_{\mathrm{OC}}$ was measured under simulated solar illumination for both $\mathrm{ZnPc}: \mathrm{C}_{60} \quad\left(V_{0 c}=0.56 \mathrm{~V}\right)$ and $\mathrm{F}_{4} \mathrm{ZnPc}: \mathrm{C}_{60}$ $\left(V_{\mathrm{OC}}=0.73 \mathrm{~V}\right)$ with the latter being $0.17 \mathrm{~V}$ higher.

The conversion of the lowest energy singlet excited state on the donor to a freeelectron-hole pair with a chemical potential $e V_{O C}$ occurs at the cost of $\Delta E_{\text {loss, }}$ given by

$$
\Delta E_{\mathrm{loss}}=E_{\mathrm{opt}}-e V_{\mathrm{OC}}
$$

The associated voltages losses $\Delta V_{\text {loss }}=\Delta \mathrm{E}_{\text {loss }} / \mathrm{e}$ are $0.97 \mathrm{~V}$ for $\mathrm{ZnPc:} \mathrm{C}_{60}$ and $0.79 \mathrm{~V}$ for $\mathrm{F}_{4}-\mathrm{ZnPc}: \mathrm{C}_{60}$. The lower loss for $\mathrm{F}_{4}-\mathrm{ZnPc}: \mathrm{C}_{60}$ is largely due to a reduced energy loss in the charge-transfer process, 
converting a relaxed phthalocyanine singlet exciton to a relaxed CT state. ignores exciton binding and polarization energies. Indeed, in the initial photo-induced electron transfer event, donor (or acceptor) excitons, with minimum energy $\left(E_{\mathrm{opt}}\right)$ are converted to CT states, with
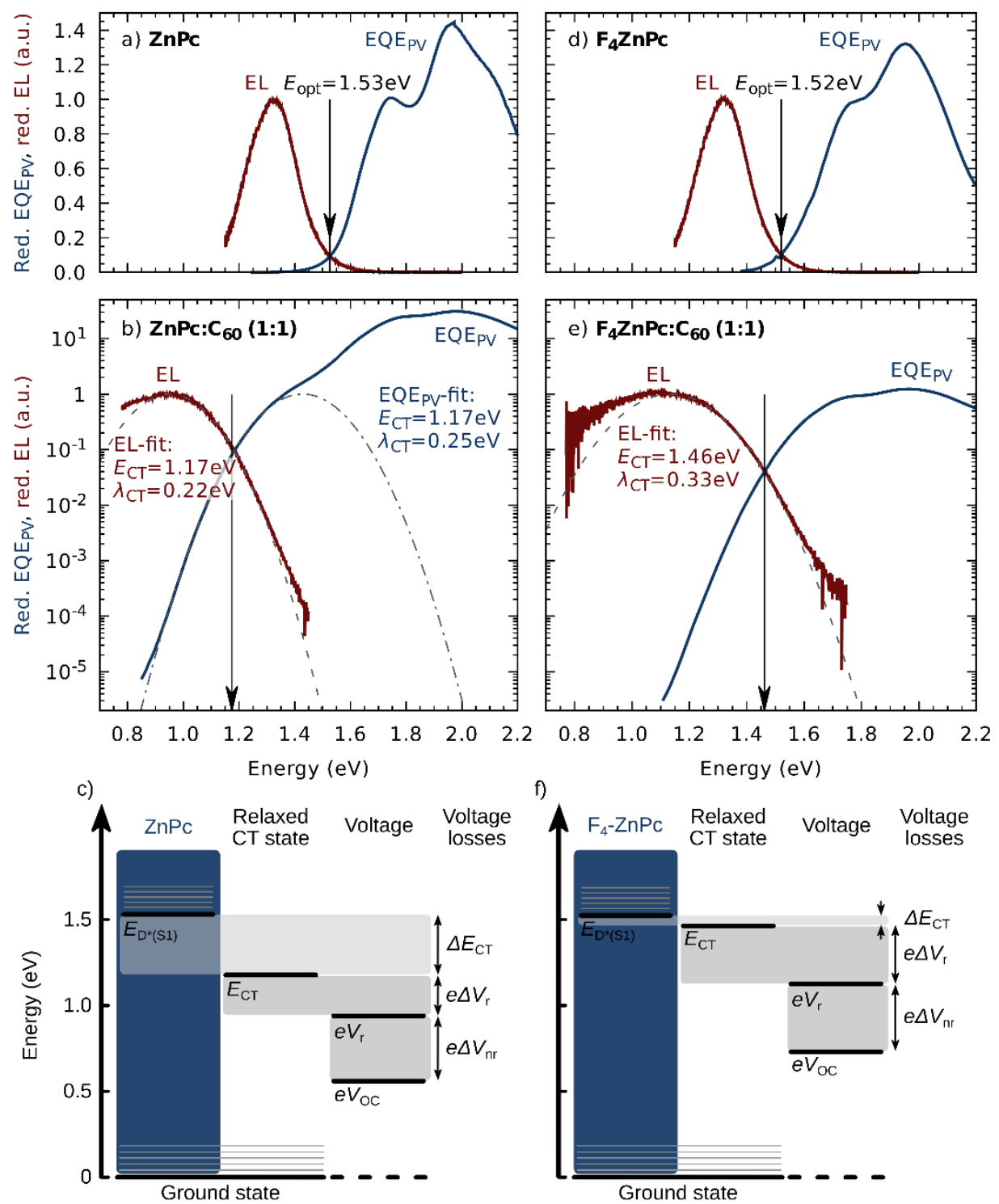

energy $E_{\mathrm{CT}}$. Seeing this process as free electrons (holes) in the LUMO(D) (HOMO(A)), converted to free electrons (holes) in the LUMO(A) (LUMO(D)) would certainly be wrong.

The chemical potential at open-circuit conditions, $e V_{\mathrm{OC}}$ is lower as compared to $E_{\mathrm{CT}}$ due to recombination of free charge carriers.

$$
\Delta V_{\mathrm{rec}}=\frac{1}{e} E_{\mathrm{CT}}-V_{\mathrm{OC}}
$$

For $\mathrm{ZnPc}: \mathrm{C}_{60}$, the recombination loss $\Delta V_{\text {rec }}$ is $0.61 \mathrm{~V}$ while for $\mathrm{F}_{4} \mathrm{ZnPc}: \mathrm{C}_{60}$ the total voltage loss is almost fully due to recombination. determining the "driving force" for charge transfer as simply taking the LUMO(D)-LUMO(A) or HOMO(A)-HOMO(D) difference, which

Figure 3: Examples of determining the $E_{o p t}$ and $E_{c t}$ for 2 different OSCs: (a) neat ZnPc, (b) ZnPc: $\mathrm{C}_{60}$ and (d) neat $\mathrm{F}_{4}-\mathrm{ZnPc}$, (e) $\mathrm{F}_{4}-\mathrm{ZnPc}: \mathrm{C}_{60}$. In every sub-figure, the black arrow shows the position of the crossing point between reduced and normalized EQEPv and EL spectra. (c) and (f) summarize the voltage losses in the two exemplary devices. The $E_{\mathrm{opt}}$ of the donor and $E_{\mathrm{Cr}}$ of the blend are obtained as described in the main text, $V_{\text {rad }}$ is calculated from the EQEpv spectra, and $V_{\text {oc }}$ is measured at 1 sun illumination for the corresponding device. Further details on the voltage losses are given in the main text. 
In general, recombination losses often comprise a substantial fraction of the total voltage losses in OSC and are often found to be around $0.6 \mathrm{~V}$, when $V_{\mathrm{OC}}$ is measured at room temperature and under 1 sun illumination. ${ }^{10,11}$

To understand the origin of the recombination caused voltage losses in more detail it is useful to consider the influence of radiative and non-radiative recombination on $V_{\text {OC }}$ separately. When only radiative recombination would occur, $V_{O C}$ would reach its upper limit, the socalled $V_{r}$, which can be calculated as: ${ }^{12,13}$

$$
V_{\mathrm{r}}=\frac{k_{\mathrm{B}} T}{q} \ln \frac{J_{S C}}{J_{0}^{r}}
$$

where $J_{\text {SC }}$ is the short-circuit current density obtained by integrating the product of the EQE $E_{P V}$ spectrum and the solar AM1.5g spectrum, and $J_{0} r$ is the radiative limit of the dark current, obtained by integrating the product of the EQE spectrum at room temperature. More details on this procedure, which assumes thermal equilibrium between $\mathrm{CT}$ states and free carriers, can be found in ref. 13 .

Voltage losses due to radiative recombination, $\Delta V_{\mathrm{r}}$ are given by

$$
\Delta V_{\mathrm{r}}=\frac{1}{e} E_{\mathrm{CT}}-V_{\mathrm{r}}
$$

They are in a sense fundamental, because they are a direct consequence of the fact that OSCs absorb light. ${ }^{14}$ From the available sensitively measured EQEpv spectra we calculate a $V_{r}$ of $0.93 \mathrm{~V}$ for $\mathrm{ZnPc}: \mathrm{C}_{60}$ and $a V_{\mathrm{r}}$ of $1.12 \mathrm{~V}$ for $\mathrm{F}_{4} \mathrm{ZnPc}_{\mathrm{n}} \mathrm{C}_{60}$, corresponding to $\Delta V_{\mathrm{r}}=0.24 \mathrm{~V}$ and $\Delta V_{\mathrm{r}}=0.34 \mathrm{~V}$, respectively. The latter radiative losses are higher due to stronger absorption of the radiatively recombining species in $\mathrm{F}_{4} \mathrm{ZnPc}: \mathrm{C}_{60}$.

The difference between the radiative limit of the $V_{\mathrm{OC}}$, the $V_{r}$, and the in reality measured $V_{\mathrm{OC}}$ corresponds to non-radiative voltage losses, $\Delta \mathrm{V}_{\mathrm{nr}}$

$$
\Delta V_{\mathrm{nr}}=V_{\mathrm{r}}-V_{\mathrm{OC}}
$$

They are due to non-radiative decay processes. It has been shown theoretically and experimentally that:

$$
\Delta V_{\mathrm{nr}}=\frac{k_{\mathrm{B}} T}{e} \ln \left(\mathrm{EQE}_{\mathrm{EL}}^{-1}\right)
$$

With $\mathrm{EQE}_{\mathrm{EL}}$ being the quantum yield of radiative decay, which is the ratio of the radiative recombination rate to the total sum of radiative and non-radiative recombination rate. To determine $\Delta \mathrm{V}_{\mathrm{nr}}$ via a measurement of $\mathrm{EQE}_{\mathrm{EL}}$ one should take care that the applied injection current is low, so that quasi-equilibrium conditions are ensured and the charge density in the device corresponds to that under solar conditions. For $\mathrm{ZnPc}: \mathrm{C}_{60}$ and $\mathrm{F}_{4} \mathrm{ZnPc}: \mathrm{C}_{60}$ these $\Delta \mathrm{V}_{\mathrm{nr}}$ are $0.38 \mathrm{~V}$ and $0.39 \mathrm{~V}$, respectively. In both cases, this is a substantial part of the total recombination losses, corresponding to an $\mathrm{EQE}_{\mathrm{EL}}$ of about $3 \times 10^{-7}$, which is a low value as compared to other photovoltaic technologies. The resulting large non-radiative voltage losses have been proposed to be intrinsic for fullerene OSCs, limiting their maximum achievable power conversion efficiency. ${ }^{11}$

\section{Temperature and illumination intensity dependence of the voltage losses}

For an analysis of the voltage losses, one should keep in mind that $V_{\mathrm{OC}}$ is temperature and illumination intensity dependent. Most expressions for $V_{\mathrm{OC}}$ are of the general form: ${ }^{15}$

$$
V_{\mathrm{OC}}=V_{0}-\beta T \ln \left(J_{\mathrm{ph}}\right)
$$

Hereby is $J_{\mathrm{ph}}$ the photocurrent density and $\beta$, a temperature and light intensity independent parameter. The extrapolation of temperature dependent $V_{\mathrm{OC}}$ measurements to $0 \mathrm{~K}$ leads to $V_{0}$ and delivers $\beta$, which depends on the details of the free carrier recombination processes.

For OSCS, $V_{0}$ has been found to correspond to $E_{\mathrm{CT}}$, rather than $\operatorname{HOMO}(D)-\operatorname{LUMO}(A) \cdot{ }^{13,16}$ It has been shown that this is due to the fact that the $\mathrm{CT}$ states are in thermal equilibrium with the free charge carriers. ${ }^{17,18}$ As a result, the total recombination losses $\Delta V_{\text {rec, }}$ radiative and non-radiative, are temperature dependent. Besides the optical method described above, temperature dependent measurements of $V_{O C}$ therefore provide and an alternative method to determine $E_{\mathrm{cT}}$ and the recombination losses.

Even when static disorder is present, the absorption and emission tails which are already broadened by electron phonon coupling are additionally broadened by the site energy spread. When fitting EQEPV and EL with equations (1) and (2), this will result in a temperature dependent $E_{\mathrm{CT}}$. However, the extrapolation of $V_{\mathrm{OC}}$ to $0 \mathrm{~K}$ will still correspond to the extrapolation of $E_{\mathrm{CT}}$ to $0 \mathrm{~K} .{ }^{17}$ Therefore, the optical method of determining $E_{C T}$ and the temperature dependent $V_{\mathrm{OC}}$ method are consistent with each other. ${ }^{13}$

\section{Minimizing energy losses for strongly absorbed photons}

To finalize this perspective, we summarize some of the characteristics which benefit low-voltage-loss OSCs. As exemplified by the $\mathrm{ZnPc}: \mathrm{C}_{60}$ and $\mathrm{F}_{4}-\mathrm{ZnPc}: \mathrm{C}_{60}$ systems, increasing $E_{\mathrm{CT}}$ to minimize 
a)

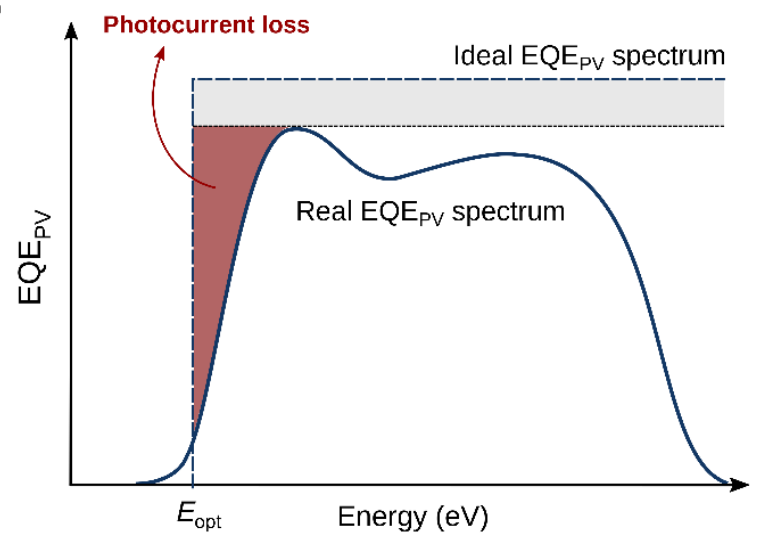

b)

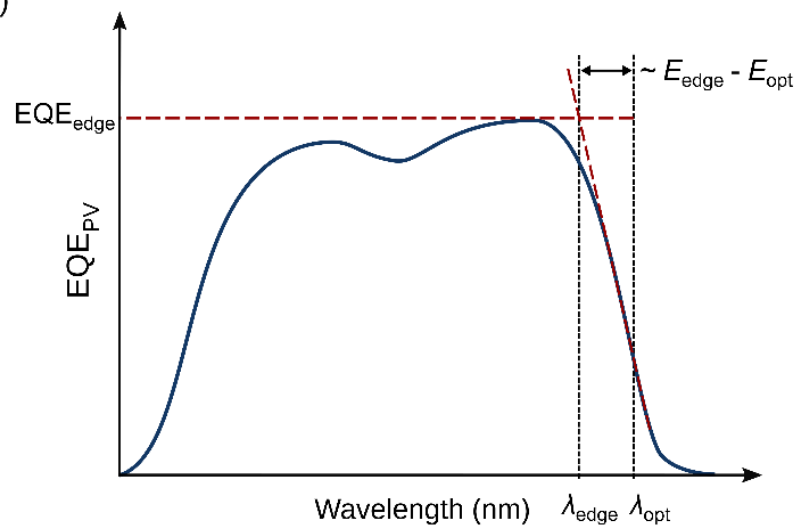

Figure 4: (a) An ideal step-wise EQEpv spectrum and an exemplary real EQEpv spectrum, exhibiting a shallow absorption edge, for a hypothetical OSC. Optical and transport losses reduce the maximum obtainable EQE $E_{p v}$ of solar cells (grey area). The shallow absorption edge induces low EQE just above $E_{\text {opt. }}$ For a steep absorption edge, this photocurrent loss is minimized. (b) Graphical determination of $\lambda_{\text {edge }}$ as the intersection of the extrapolated linear part of the absorption edge, and the isoline passing through the EQE peak at low-energy edge (EQEedge) of the EQEpv spectrum. $E_{\text {edge }}$ Corresponds to the energy of the absorbed low-energy photons which highly contribute to the device's photocurrent, and is obtained as $1240 / \lambda_{\text {edge }}(\mathrm{nm}$ ). Considering energy losses from $e V_{o c}$ to $E_{\text {edge }}$ takes into account the steepness of the absorption edge, and promotes solar cells exhibiting $E_{\text {edge }}$ close to $E_{\text {opt. }}$ Here, $\lambda_{\text {opt }}$ is the wavelength associated with $E_{\mathrm{opt}}$ as $\lambda_{\mathrm{opt}}=1240 / E_{\mathrm{opt}}(\mathrm{eV})$. With known $E_{\mathrm{opt}}$, the offset $E_{\mathrm{edge}}-E_{\mathrm{opt}}$ is a measure of the absorption edge steepness. Efficient solar cell performance requires the lowest $E_{\text {edge }}-e V_{O c}$ difference at the highest possible $E_{\text {Q }} E_{\text {edge }}$.

$\Delta E_{C T}$ can be done by chemical design, controlling the frontier energy levels of donor and acceptor. For several new donor-acceptor combinations for OSCs, $\Delta E_{\mathrm{CT}}$ is vanishingly small, while still high $\mathrm{EQE}_{\mathrm{PV}}$ values can be achieved. ${ }^{5,19,20}$ In those cases, the recombination losses $\Delta V_{\text {rec }}$ are the dominant ones. Up to now, only few strategies to suppress these losses have been proposed, including the suppression of the donor-acceptor interfacial area to reduce $\Delta V_{r},{ }^{21-23}$ and the use of a cascaded device architecture, suppressing partly $\Delta V_{\mathrm{nr}}{ }^{5}$

It is however important to note that even if the voltage losses $E_{\text {opt }^{-}}$ $e V_{\text {oc }}$ are minimized, this does not necessarily mean that the solar cell will be highly efficient. Complementary to a high $V_{O C}$, a high PCE requires also high photocurrent and FF. For this to be the case, charge generation and extraction has to be efficient at low $\Delta E_{\mathrm{CT}}$ and the EQE $E_{P V}$ needs to be high (near unity) for all photons with an energy higher than $E_{\text {opt. }}$. However, the shallow absorption edge of organic materials results in low $E Q E_{\mathrm{PV}}$ for photon energies close to $E_{\mathrm{opt}}$ (see Figure 4a).

We have recently introduced a metric which takes this into account and considers voltage losses for strongly absorbed photons, at the low-energy tail of the EQE $E_{P V}$ spectrum, as the difference $E_{\text {edge }}-e V_{\text {oc. }}{ }^{5}$ Hereby $E_{\text {edge }}$ is defined as illustrated in Fig $4 \mathrm{~b}$. For efficient solar cells, a low difference $E_{\text {edge }}-e V_{O C}$ at very high $\mathrm{EQE}_{\text {edge }}$ is required. Energy losses related to the steepness of the main absorber's absorption edge are quantified by $E_{\text {edge }}-E_{\text {opt, }}$ implying that for OSCs exhibiting the desired steep absorption edge, the $E_{\text {edge }}-E_{\text {opt }}$ offset will be minimal. To keep these losses low, Figure 2 shows that small lowfrequency relaxation energies of the neat material excitons are required.

The determination of the voltage losses for strongly absorbed photons with this metric requires only standard $E_{Q E_{P V}}$ and $J-V$ measurements. This has the advantage that basically every photovoltaic device whose EQEpv spectrum and $J-V$ curve are known can be compared in terms of voltage losses. However, for a deeper physical insight and identification of the voltage limiting factors, exact determinations of $E_{\mathrm{opt}}$ and $E_{\mathrm{CT}}$ are essential.

\section{Concluding statement}

More systematic analysis of voltage losses of future donor-acceptor combinations for organic photovoltaics is required for a rigorous comparison between results and materials from different research groups. With this perspective, we encourage the reader not to use ill-defined absorption onsets, at which virtually no photons are absorbed, as reference point for voltage losses. Nor do we promote the use of HOMO and LUMO energy levels to determine the relevant energies of excited states in OSCs. The optical measurements described above, or temperature dependent measurements of $V_{O C}$ contain much more useful and precise information. Finally, we would also like to encourage researchers working on the minimization of voltage losses in OSCs, to accompany claims of low voltage losses with a measurement of the EQE $E_{\mathrm{EL}}$ (measured at an applied voltage of $V_{\mathrm{OC}}$ ), since solar cells with truly low voltage losses will also be efficient LEDs.

\section{Conflicts of interest}

There are no conflicts to declare.

\section{Acknowledgements}


This work received funding from the German Federal Ministry for Education and Research (BMBF) through the InnoProfile Projekt "Organische p-i-n Bauelemente 2.2" (03IPT602X).

\section{Notes and references}

Y. Cui, H. Yao, B. Gao, Y. Qin, S. Zhang, B. Yang, C. He, B. Xu and J. Hou, J. Am. Chem. Soc., 2017, jacs.7b01493.

W. Zhao, S. Li, H. Yao, S. Zhang, Y. Zhang, B. Yang and J. Hou, J. Am. Chem. Soc., 2017, 139, 7148-7151. X. Guo, N. Zhou, S. J. Lou, J. Smith, D. B. Tice, J. W. Hennek, R. P. Ortiz, J. T. L. Navarrete, S. Li, J. Strzalka, L. X. Chen, R. P. H. Chang, A. Facchetti and T. J. Marks, Nat. Photonics, 2013, 7, 825-833. S. Li, W. Liu, C.-Z. Li, M. Shi and H. Chen, Small, 2017, 1701120, 1701120. V. C. Nikolis, J. Benduhn, F. Holzmueller, F. Piersimoni, M. Lau, O. Zeika, D. Neher, C. Koerner, D. Spoltore and K. Vandewal, Adv. Energy Mater., 2017, 1700855.

6 J.-L. Bredas, Mater. Horiz., 2014, 1, 17-19.

7 A. Kohler and H. Bassler, Electronic Processes in Organic Semiconductors: An Introduction, Wiley-VCH Verlag $\mathrm{GmbH}$ \& Co. KGaA, Weinheim, Germany, 2015, vol. 1.

R. A. Marcus, J. Phys. Chem, 1989, 93, 3078-3086.

9 K. Vandewal, S. Albrecht, E. T. Hoke, K. R. Graham, J. Widmer, J. D. Douglas, M. Schubert, W. R. Mateker, J. T. Bloking, G. F. Burkhard, A. Sellinger, J. M. J. Fréchet, A. Amassian, M. K. Riede, M. D. McGehee, D. Neher and A. Salleo, Nat. Mater., 2014, 13, 63-68.

10 K. R. Graham, P. Erwin, D. Nordlund, K. Vandewal, R. Li, G. O. Ngongang Ndjawa, E. T. Hoke, A. Salleo, M. E. Thompson, M. D. McGehee and A. Amassian, Adv. Mater., 2013, 25, 6076-6082. J. Benduhn, K. Tvingstedt, F. Piersimoni, S. Ullbrich, Y. Fan, M. Tropiano, K. A. McGarry, O. Zeika, M. K. Riede, C. J. Douglas, S. Barlow, S. R. Marder, D. Neher, D. Spoltore and K. Vandewal, Nat. Energy, 2017, 2, 17053.

12 U. Rau, Phys. Rev. B, 2007, 76, 85303.

13 K. Vandewal, K. Tvingstedt, A. Gadisa, O. Inganäs and J. V. Manca, Phys. Rev. B, 2010, 81, 1-8.

14 P. Würfel and U. Würfel, Physics of Solar Cells: From Basic Principles to Advanced Concepts, Wiley-VCH Verlag GmbH \& Co. KGaA, Weinheim, Germany, 2016. M. A. Green, Prog. Photovoltaics Res. Appl., 2003, 11, 333340. U. Hörmann, J. Kraus, M. Gruber, C. Schuhmair, T. Linderl, S. Grob, S. Kapfinger, K. Klein, M. Stutzman, H. J. Krenner and W. Brütting, Phys. Rev. $B$, , DOI:10.1103/PhysRevB.88.235307. T. M. Burke, S. Sweetnam, K. Vandewal and M. D.

K. Vandewal, Annu. Rev. Phys. Chem, 2016, 67, 113-33. N. A. Ran, J. A. Love, C. J. Takacs, A. Sadhanala, J. K. Beavers, S. D. Collins, Y. Huang, M. Wang, R. H. Friend, G. C. Bazan and T. Q. Nguyen, Adv. Mater., 2016, 28, 14821488.

J. Liu, S. Chen, D. Qian, B. Gautam, G. Yang, J. Zhao, J. Bergqvist, F. Zhang, W. Ma, H. Ade, O. Inganäs, K. Gundogdu, F. Gao and H. Yan, Nat. Energy, 2016, 1, 16089. K. Vandewal, J. Widmer, T. Heumueller, C. J. Brabec, M. D. McGehee, K. Leo, M. Riede and A. Salleo, Adv. Mater., 2014, 26, 3839-3843.

D. Credgington and J. R. Durrant, J. Phys. Chem. Lett., 2012, 3, 1465-1478.

M. A. Fusella, A. N. Brigeman, M. Welborn, G. E. Purdum, Y. Yan, R. D. Schaller, Y. L. Lin, Y.-L. Loo, T. Van Voorhis, N. C. Giebink and B. P. Rand, Adv. Energy Mater., 2017, 1701494, 1-10. McGehee, Adv. Energy Mater., 2015, 5, 1-12. 